\title{
Arterial tortuosity syndrome: A rare inherited form of connective tissue disorder with SLR2A10 gene mutation
}

\author{
*Sithija Wijesinghe ${ }^{1}$, Deepthi De Silva ${ }^{2}$, Duminda Samarasinghe ${ }^{1}$, Sunethra Irugalbandara ${ }^{3}$
}

Sri Lanka Journal of Child Health, 2022; 51(1): 142-145

DOI: http://dx.doi.org/10.4038/sljch.v51i1.10023

(Key words: Arterial tortuosity syndrome (ATS), SLC2A10 gene, Autosomal recessive, Homozygous mutation)

\section{Introduction}

Arterial tortuosity syndrome (ATS) is an extremely rare autosomal recessively inherited connective tissue disorder characterized by elongation, twisting, stenosis and aneurysm formation of large and medium size arteries 1 . The phenotype also includes characteristic facial features, skeletal involvement, skin laxity, hernias, intestinal and genitourinary involvement ${ }^{2}$. Histological findings, including disruption of elastic fibres of the media and fragmentation of the internal elastic membrane of large and medium size arteries have been described ${ }^{2}$.

ATS is caused by homozygous mutations in SLC2A10 gene which is autosomal recessively inherited and located in the long arm (q) of chromosome $20(20 \mathrm{q} 13.1)^{2}$. The gene encodes GLUT 10, a facilitated glucose transporter. GLUT 10 is expressed in mitochondria and endoplasmic reticulum membranes and may act by scavenging of free radicles in mitochondria and collagen maturation in the endoplasmic reticulum but the mechanism of its deficiency in causing the histological changes or the arterial vascular anomalies is not known. ATS can lead to lifethreatening vascular complications such as rupture, dissection and ischaemic insult due to blockage of blood supply to vital organs and was considered a potentially fatal condition in early infancy ${ }^{1}$. Current evidence suggests that the occurrence of complications is variable and less severe $^{2,6}$. Life expectancy is longer and the prevalence of sudden vascular events are rare in adolescence

${ }^{1}$ Lady Ridgeway Hospital for Children, Colombo, Sri Lanka, ${ }^{2}$ Faculty of Medicine, University of Kelaniya, Sri Lanka, ${ }^{3}$ Sirimavo Bandaranayake Children's Hospital, Peradeniya, Sri Lanka *Correspondence: sithijapiumi@gmail.com

$$
\text { (iD) }
$$

https//orcid.org/ 0000 - 0001- 9432 - 0862

(Received on 19 November 2020: Accepted after revision on 22 January 2021)

The authors declare that there are no conflicts of interest

Personal funding was used for the project.

Open Access Article published under the Creative

Commons Attribution CC-BY (c) (i) License or in adults ${ }^{2}$ than previously described. ATS does not show gender predilection ${ }^{2}$. The prevalence or incidence is unknown ${ }^{2}$. Approximately 100 cases have been reported so far across the world ${ }^{2}$.

\section{Case report}

The proband is a two year ten-month-old boy, the $3^{\text {rd }}$ child of healthy non-consanguineous parents born following uneventful pregnancy and delivery. His growth and psychomotor development were age appropriate. An incidental cardiac murmur was detected following admission to a local hospital with a lower respiratory tract infection and he was subsequently referred to the Lady Ridgway Hospital for specialised cardiology evaluation.

He had an uncomplicated neonatal period and infancy. There was no known family history of sudden cardiac death or vascular disease. On clinical examination, he was noted to have midfacial hypoplasia, blepharophimosis, down slanting palpebral fissures and Harrison's sulci.

His initial echocardiographic evaluation revealed a dilated aortic root, main pulmonary artery (MPA) with proximal branch pulmonary artery (BPA) stenosis and Grade 2 aortic regurgitation.

Cardiac catheterization was performed to visualize the aortic and pulmonary vasculature (Figures 1-6).

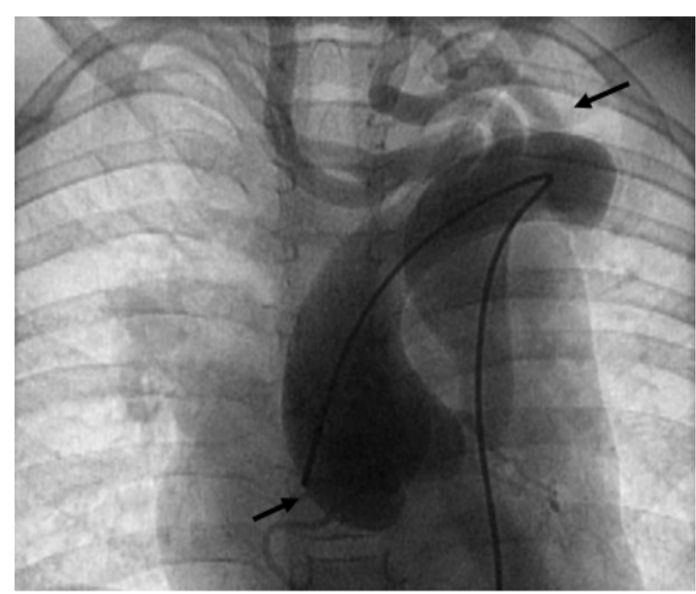

Figure 1: Aortic angiogram showing a dilated aortic root (arrowed), and tortuosity of main head and neck vessels 


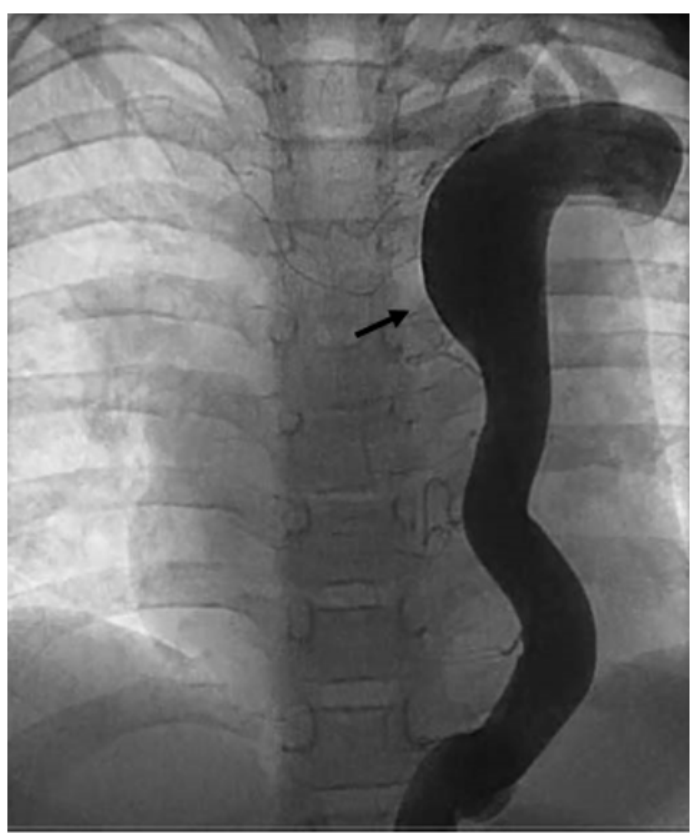

Figure 2: Descending aortic angiogram showing aneurysmal dilatation (arrowed) and tortuosity

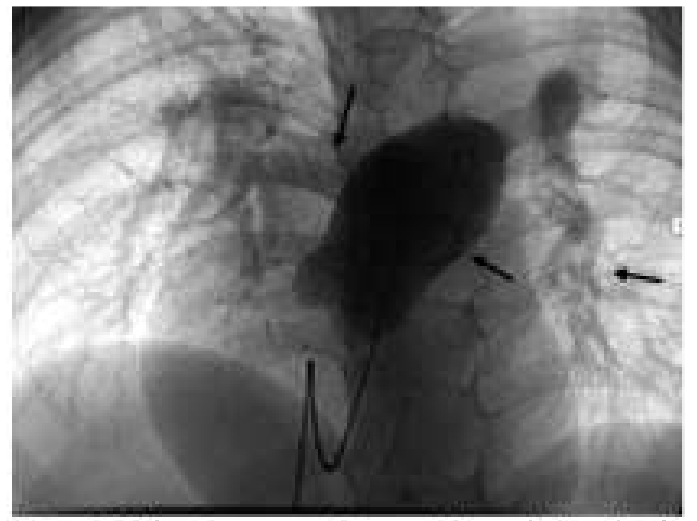

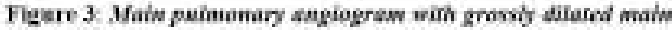

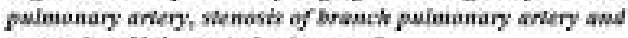
apraasiby of lobar arieries (arrowet)

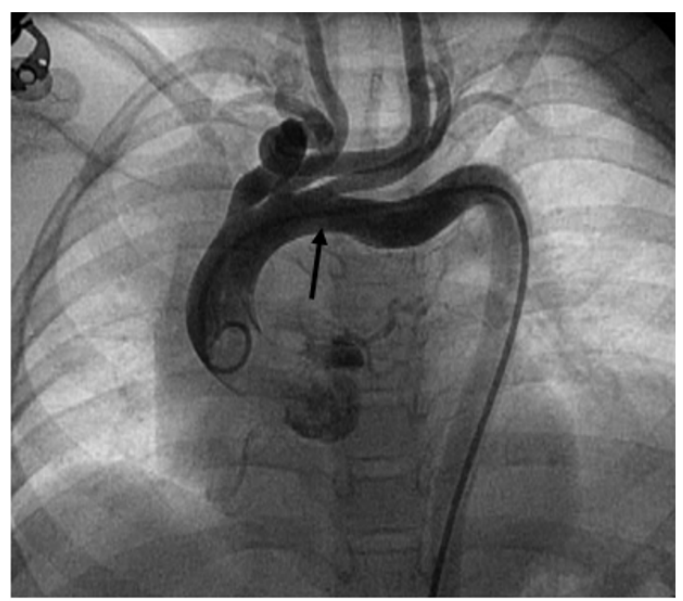

Figure 4: Aortic arch angiogram showing elongated aortic arch (arrowed) and extensive tortuosity brachiocephalic vessels

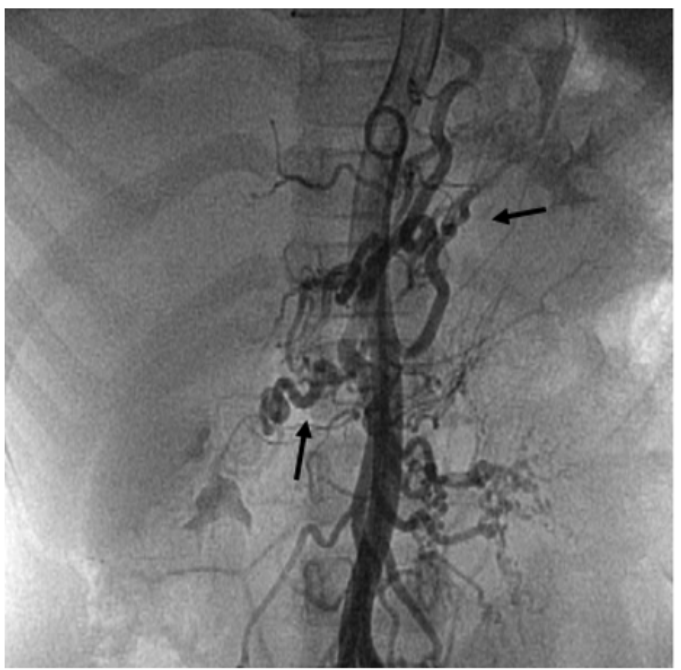

Figure 5: Descending aortic angiogram showing tortuosity of mesenteric and renal vessels (arrowed)

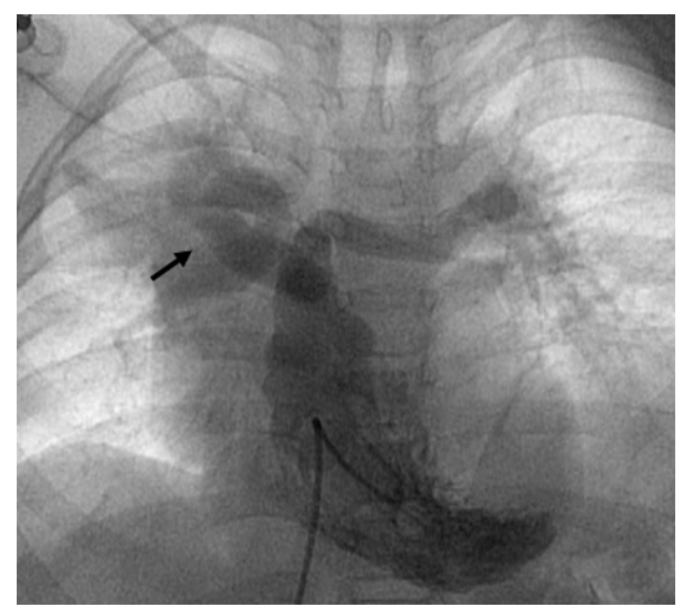

Figure 6: Main pulmonary artery angiogram depicting the dilated main pulmonary artery and tortuosity of branch pulmonary arteries of sister

Aortic angiogram revealed a dilated aortic root, left sided elongated and tortuous aortic arch and brachiocephalic vessels (Figures 1 and 4). The descending thoracic aorta demonstrated aneurysmal dilatation. The abdominal aorta, mesenteric and renal arteries also showed evidence of tortuosity (Figures 2 and 6). Pulmonary angiogram showed dilated MPA with bilateral proximal BPA stenosis. The lobar and segmental pulmonary arteries also illustrate abnormal tortuosity (Figures 3 and 5).

His six-year-old sister had a history of hiatal hernia in the neonatal period for which she had surgery during the first week of life. On examination, she showed facial similarities to her bother with mild skin laxity and hypermobile joints. Her echocardiogram showed dilated pulmonary arteries and dilated aortic root. Aortic and pulmonary angiograms were performed to delineate anatomy of the arterial system (Figures 4, 5 and 6) and 
confirmed the echocardiogram findings. Ophthalmological examination was normal. Mutation testing was performed using genomic DNA extracted from blood in a specialised laboratory using previously described methods ${ }^{7}$. This confirmed a homozygous SLC2A10 gene in both affected siblings but not their unaffected carrier parents. Another sibling was neither clinically affected nor proven to share the gene mutations.

\section{Discussion}

ATS is an extremely rare autosomal recessively inherited multisystem, connective tissue disorder with characteristic vascular involvement ${ }^{1}$. The disease can manifest in the neonatal period ${ }^{1,3}$. The described features of ATS include characteristic long face, hypertelorism, blepharophimosis, long philtrum, down slanting palpebral fissures, micrognathia and beaked nose and they tend to look older than their age ${ }^{1,2}$. Keratoconus is seen less commonly ${ }^{2}$.

Skin manifestations include soft, doughy skin, hyper-extensibility of the skin but no increased bruisability. The bone and skeletal manifestations are arachnodactyly, high arched palate, pectus carinatum, joint contractures and joint laxity. The cardiovascular manifestations are the main cause of mortality ${ }^{6}$. The cardiovascular manifestations include ventricular hypertrophy, hypertension, pulmonary artery stenosis, aortic stenosis, arterial tortuosity and elongated arteries (large and medium-sized arteries including aorta) ${ }^{3}$. Further, hugely dilated aortic root and ascending aorta, tortuosity of arch, dilated MPA with proximal stenosis of BPA were described $^{4}$. Tortuosity of the aorta or large arteries were invariably present ${ }^{5}$. The heterozygous carriers do not show arterial tortuosity or other phenotypic characteristics $^{5}$. ATS is also associated with hernia including hiatal, diaphragmatic, sliding and gastric hernia $^{3}$. In addition, diverticulitis, bowel necrosis due to thrombosis, ischaemic stroke, mental retardation and hypotonia are also described in association with ATS and these manifestations are less common ${ }^{2}$. Although the symptoms of ATS are well defined, many details of this syndrome are not fully established due to rarity of the disease and lack of large clinical studies ${ }^{2}$.

Both our patients showed most of the common phenotypic features. They did not have diverticulitis, keratoconus, hypotonia or mental retardation. Our patients are now 13 years old and 9 years old and do not have any vascular emergencies. The occurrence of sudden lifethreatening vascular complications beyond infancy and early childhood is similar to the general population ${ }^{2}$. Parents and the eldest sister were screened with history, clinical examination and echocardiography but showed no phenotypic features of ATS. The diagnosis of ATS is based on clinical evaluation directed at identification of characteristic symptoms and signs ${ }^{2}$. The specialised investigations helpful in the diagnosis of ATS are echocardiography, magnetic resonance angiography (MRA) and computed tomography $(\mathrm{CT})^{2}$. All these imaging modalities are useful in establishing the vascular phenotypic features of the disease $^{2,3}$. However, the confirmation of ATS is based on the demonstration of SLC2A10 gene mutation $^{2}$. Twenty-five mutations are described up to now including missense substitutions, nonsense nucleotide changes and deletions ${ }^{6}$. Both our patients had SLR2A10 gene mutation due to deletions.

Phenotype of ATS shows significant overlap with other syndromes and connective tissue disorders like Loeys-Dietz syndrome, Ehlers-Danlos syndrome, Marfan syndrome and autosomal recessive cutis laxa type $1^{2,6}$. Each of these conditions should considered in differential diagnosis $^{6}$. The management of ATS is multidisciplinary and includes treatment of manifestations, surveillance, lifestyle modifications and genetic counselling for individuals who are at risk $^{2,6}$. Treatment is with beta blockers, angiotensin converting enzymes (ACE) inhibitors and angiotensin 11 receptor blockers to reduce the haemodynamic stress on vessel wall and hence reduce the risk of sudden vascular events ${ }^{6}$. The treatment should be cautious and the effectiveness is not established ${ }^{6}$. Localised aneurysms and stenosis are treated surgically ${ }^{6}$. Hernias need mesh repair as it can reoccur even following the primary repair ${ }^{6}$ Ophthalmology and orthopaedic complications need specialised management ${ }^{6}$.

ATS patients need routine follow up for cardiovascular manifestations including hypertension, pulmonary hypertension and ventricular dysfunction with echocardiograms and angiograms performed at regular intervals ${ }^{2,6}$. In addition, affected individuals need regular pulmonology, ophthalmology, orthopaedic and orthodontic evaluations ${ }^{2,6}$. Affected individual should not engage in contact sports, competitive sports or isometric exercises ${ }^{6}$. They should avoid routine use of decongestants, tobacco and suntanning ${ }^{6}$. Genetic counselling is recommended to siblings and high-risk relatives of affected individuals ${ }^{2,6}$.

SLR2A10 gene encodes GLUT10 ${ }^{2}$. GLUT10 is a protein with 541 amino acids and a member of the class III facilitative glucose transporter family ${ }^{2}$. However, the exact molecular pathophysiology of how defective GLUT10 causes ATS is not fully 
described yet ${ }^{2,6}$. The SLR2A10 lies within the 20q12-13 region which linked to Type 11 diabetes mellitus ${ }^{6}$. Current evidence does not indicate increased susceptibility to overt diabetes mellitus in $\mathrm{ATS}^{5,6}$. Our patient's glucose tolerance was normal.

ATS was first described by Ertrugal in $1967^{6}$. In ATS the clinical manifestation and presentation is diverse due to its multisystem involvement and vascular manifestations ${ }^{2}$. Early identification is very important considering the limited treatment options, surveillance for manifestations and complications, family counselling and genetic counselling ${ }^{1,2,5}$. The pregnancy related issues of this rare entity are not yet described ${ }^{6}$. Umbilical and hiatal and inguinal hernias, cutis laxa and unexplained respiratory distress in the neonatal period are common and need cardiovascular evaluation in view of early identification of this rare entity ${ }^{2,5,6}$.

The severe vascular complications include early aggressive aortic root aneurysms, neonatal intracranial bleeding, ischaemic stroke and gastric perforation ${ }^{6}$. No reports document vascular dissections or ruptures among the existing literature beyond the neonatal period ${ }^{2,6}$. The clinical spectrum of this rare vascular disease warrants diagnostic workup for infantile respiratory distress syndrome (IRDS), hernias (umbilical, hiatal, inguinal and diaphragmatic) and cutis laxa ${ }^{5}$. ATS children need close monitoring of the aortic root early in life, and extensive vascular imaging afterwards ${ }^{2,6}$.

\section{References}

1. Genetics Home Reference. NIH (US National Library of Medicine). Available from: https:/ghr.nlm.nih.gov/condition/arterialtortuosity-syndrome

2. National Organization of Rare Disease. Available from: https://rare-diseases/arterial-tortuositysyndrome
3. MalaCards- Human Disease Database. Available from:

https://www.malacards.org/card/arterialtortuosity-syndrome

4. Satish G, Sheela Nampoothiri S, Kappanayil M. Arterial tortuosity syndrome: Phenotypic features and cardiovascular manifestations. Circulation 2008; 117(23): 477-8. https://doi.org/10.1161/CIRCULATIONA HA.107.739839

PMid: 18541745

5. Callewaert BL, Willaert A, KerstjensFrederikse WS, De Backer J, Devriendt K, Albrecht $\mathrm{B}$, et al. Arterial tortuosity syndrome: Clinical and molecular findings in 12 newly diagnosed families. Human Mutation Variation, Informatics and Disease 2008; 29(1): 150-8.

https://doi.org/10.1002/humu.20623

PMid: 17935213

6. Callewaert B, De Paepe A, Coucke P. Gene Reviews. Available from:

https://www.ncbi.nlm.nih.gov/books/NBK 253404

7. Ritelli M, Chiarelli N, Dordoni C, Refflo E, Venturini M, Quinzani S, et al. Arterial tortuosity syndrome. BMC Medical Genetics 2014; 15; 122. https://doi.org/10.1186/s12881-014-01225

PMid: 25373504 PMCid: PMC4412100 